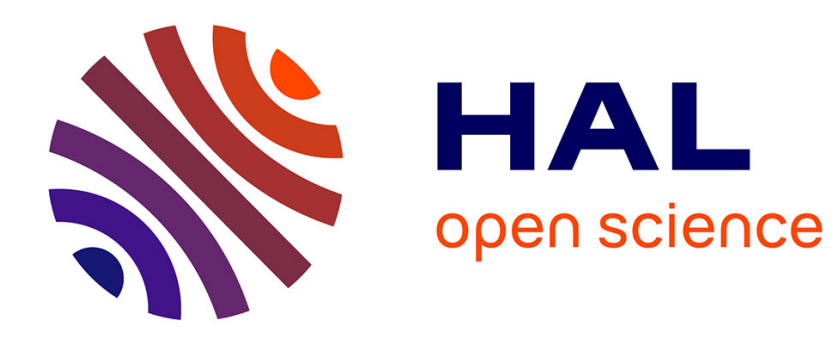

\title{
Imperfections et lacunes des passifs du français
}

Claude Muller

\section{To cite this version:}

Claude Muller. Imperfections et lacunes des passifs du français. Études de linguistique appliquée: revue de didactologie des langues-cultures, 2017, 187, pp.283-296. hal-02123293

\section{HAL Id: hal-02123293 \\ https://hal.science/hal-02123293}

Submitted on 7 May 2019

HAL is a multi-disciplinary open access archive for the deposit and dissemination of scientific research documents, whether they are published or not. The documents may come from teaching and research institutions in France or abroad, or from public or private research centers.
L'archive ouverte pluridisciplinaire HAL, est destinée au dépôt et à la diffusion de documents scientifiques de niveau recherche, publiés ou non, émanant des établissements d'enseignement et de recherche français ou étrangers, des laboratoires publics ou privés. 
Claude Muller

\section{IMPERFECTIONS ET LACUNES DES PASSIFS DU FRANÇAIS}

publié dans Etudes de linguistique appliquée, 187, juillet-septembre 2017, 283-296.

Résumé : On passe en revue les formes verbales (au sens large) produisant des effets de diathèse passive. Au passif par auxiliaire, qui peut se combiner à l'impersonnel, s'ajoutent des passifs pronominaux et de nombreuses autres constructions moins nettement grammaticalisées. Aucun de ces outils grammaticaux ne procure une correspondance simple et évidente permettant de passer de l'actif au passif, la principale déficience du français résidant dans la difficulté du verbe être à exprimer des actions en cours.

Il n'y a jamais de correspondance exacte entre la construction active et la construction passive ${ }^{1}$ : cette dernière, dans une langue accusative comme le français, est toujours marquée, parce qu'elle s'affranchit de la correspondance naturelle qui existe dans ce type de langue entre le rôle d'agent et la fonction de sujet.

On ne passera pas en revue dans leur totalité les insuffisances et blocages divers des constructions passives du français. L'exposé sera consacré aux insuffisances ou blocages du marquage passif dans le verbe, ainsi qu'aux variantes de passif qui en découlent.

\section{DIATHESES ET VOIX OU CONSTRUCTIONS.}

Il y a deux façons d'aborder le passif: en termes de visée informative et sémantique, le passif relève de la hiérarchie informative des rôles actanciels. Ces rôles, ce sont les relations qui associent chaque actant à son prédicat, avec une hiérarchie fonctionnelle qui distingue selon leur rang celui qui est sujet, celui qui est objet direct, et ainsi de suite. Il y a une "diathèse" ("disposition") de base pour chaque prédicat, dont le modèle prototypique est la diathèse personnelle active. Des modifications "volontaires" sont possibles, qui relèvent de la hiérarchie informative. Les diathèses passives visent, non pas à promouvoir systématiquement le $2 \mathrm{e}$ actant en sujet, mais à écarter (sans nécessairement le supprimer) le ler actant (celui qui correspond à l'agent des verbes d'action), soit en reprenant l'expression de Shibatani (1985): "defocussing the agent", ou celle de Gaatone (1998): le support n'est pas le 1er argument. Cela n'aboutit pas nécessairement à l'utilisation comme sujet du 2e actant: le passif peut se combiner à l'impersonnel, qui relève aussi de la diathèse: laisser vacante la position principale de topicalisation, sans sujet nominal, c'est privilégier l'action verbale comme telle.

\footnotetext{
${ }^{1}$ Pour le français, on peut se reporter à Gaatone (1998), Schøsler (2000). Pour mes analyses du passif, Muller (2000), (2008).
} 
L'autre façon d'aborder le passif, qui est complémentaire avec la première, a trait aux modifications grammaticales dans le verbe liées à la diathèse, ou à la combinaison de faits de diathèse. On parle de "voix" passive dans les grammaires pour les particularités que les choix de diathèse imposent non aux fonctions actancielles, mais à leur correspondance fonctionnelle dans le mot verbal. Le terme peut être remplacé par celui, moins connoté, de "construction" (Wilmet). La "construction" passive, associée ou non à d'autres faits de diathèse comme l'impersonnel, est limitée traditionnellement à l'utilisation d'un verbe, être, qui est orienté vers le 2e actant et supprime la transitivité. Mais si on prend en considération l'ensemble de la construction verbale, d'autres moyens grammaticaux peuvent être pris en compte, d'une part dans le mot verbal conjugué étendu (incluant les clitiques), d'autre part dans le verbe lexical, sous la forme du participe passé, et peut-être parfois de l'infinitif comme on le verra.

On va d'abord faire la liste des rôles d'actants en jeu, ainsi que des organisations sous forme hiérarchisée des diathèses, selon la répartition des fonctions, dans les constructions active (non marquée) et passive (à auxiliaire être).

- Rôles actanciels pertinents pour le français:

-action verbale (il impersonnel):

Ce rôle est mis en évidence dans les verbes impersonnels, et c'est le seul qui apparaît dans les verbes sans actant matériel, comme les verbes atmosphériques: il met au premier plan l'action verbale.

-relation 1er actant (agent) /action (sujet /verbe: il, elle):

Ce rôle associe la fonction de sujet à l'agent prototypique des verbes d'action, et c'est presque toujours le seul rôle disponible pour les verbes uniactanciels.

-relation 2e actant (patient)/ action (objet direct verbe : le):

C'est le rôle des objets dans les verbes d'action, prééminent pour le deuxième actant des verbes biactanciels.

-relation 3e actant (bénéficiaire) /action (datif/objet : lui):

C'est la troisième fonction, assimilable à un datif. Elle est peu concernée par le passif verbal en être.

-autres (circonstants, relations indirectes.

-Hiérarchie des diathèses: le signe > signale la priorité d'un rôle actanciel dans l'assignation d'une fonction grammaticale.

active personnelle: $\quad$ ler actant $>2 \mathrm{e}$ actant $>\ldots$

active impersonnelle: $\quad$ action $>1$ er actant $>(2 \mathrm{e}$ actant $)>\ldots$

passive personnelle: $\quad 2 \mathrm{e}$ actant $>\ldots . .>1 \mathrm{er}$ actant

passive impersonnelle: $\quad$ action $>2 \mathrm{e}$ actant $>\ldots>($ ler actant $)$

Comment la construction du verbe peut-elle orienter les choix de diathèse? Cela peut être obtenu en modifiant la transitivité (c'est ce qui se 
passe avec le verbe être), ou à bloquer les reflets actanciels dans l'environnement clitique: le $i l$ impersonnel bloque la réalisation d'un sujet lexical; le se bloque un actant par coréférence. Cela n'aboutit pas forcément à un résultat uniforme (voir la gamme très large des emplois du réfléchi en français ${ }^{2}$ ). L'actant empêché peut encore figurer dans la phrase, mais il est déclassé (ainsi l'agent, au lieu d'être sujet et obligatoire comme à l'actif, devient circonstanciel et facultatif: c'est le "complément d'agent" des grammaires). Enfin, ces moyens sont partiels et souvent associés, formant des diathèses mixtes.

\subsection{Un exemple: les combinaisons de l'impersonnel}

Le choix d'une construction impersonnelle (donc sans sujet lexical) est aussi en un sens un choix de construction verbale, si le clitique $i l$ y est attaché. La modification induite permet de mettre en avant la seule prédication verbale, l'ordre des actants restant inchangé, mais avec une contrainte; il y a une seule position de complémentation nominale directe du verbe, réservée à l'agent:

(1) Il mange chaque jour une dizaine de personnes dans ce restaurant

(2) *Il mange chaque jour des steaks une dizaine de personnes dans ce restaurant (N. Papon 1995: 110)

L'accusatif clitique est marginal. En voici un exemple où cohabitent l'agent des renards et l'objet vous:

(3) Il vous y guette des renards / et des loups à qui nul n'échappe (Aragon, Le voyage de Hollande, Hériau 1980)

Avec des noms, l'impersonnel actif évacue le 2e actant. Une diathèse mixte combinant impersonnel et passif existe aussi: cette fois, c'est l'objet qui figure en complément:

(4) Il est mangé chaque jour une dizaine de kilos de viande de bruf dans ce restaurant (Papon)

1.2. Les réfléchis passifs et les diathèses de promotion d'autres compléments.

Il faut ajouter à cela l'utilisation de la construction réfléchie, "voix réflexive" pour Damourette et Pichon -il s'agit aussi d'une modification du mot verbal étendu aux clitiques. Il y a deux versions qui nous concernent parmi les nombreux emplois possibles (réfléchi, réciproque, moyen, passif, antipassif). Le passif réfléchi présente aussi deux versions, l'une personnelle, l'autre impersonnelle:

-le passif réfléchi personnel:

(5) Les livres se sont vendus comme des petits pains

\footnotetext{
${ }^{2}$ Ci-dessous et plus en détail dans Muller, 2007, 2008.
} 
-l'impersonnel passif réfléchi:

(6) Il se raconte de drôles d'histoires sur son compte

A cela s'ajoutent d'autres possibilités moins grammaticalisées de diathèse:

-diathèse du 3e actant / datif, avec se voir/se faire +Inf; se trouver + Vpp

(7) Paul s'est vu octroyer un délai complémentaire (François, 2003: 284)

(8) Je me suis fait voler mon portefeuille dans le métro

(9) Je me suis trouvé contraint de sortir

-diathèse de circonstants de lieu / de temps, sans modification passive (le ler actant est objet direct fonctionnel):

(10) Saint-Malo a vu naître Chateaubriand (J. François, 2003, 282)

(11) Le 18 mai 2017 a vu quasiment disparaître le parti socialiste de la chambre des députés

\section{LE PASSIF VERBAL: PROBLEMES DUS AU VERBE ETRE.}

\subsection{Auxiliaire processif ou copule?}

Un des principaux facteurs de la difficulté d'emploi du passif tient au verbe utilisé, être. Il est auxiliaire de passif avec une interprétation particulière dans le cas des verbes d'action, que j'ai appelée "processive": la périphrase verbale décrit alors un processus en cours aux temps imperfectifs, présent et imparfait notamment. C'est seulement dans ce cas qu'on a une utilisation pleinement auxiliaire du verbe être pour signifier le passif: l'auxiliaire ne doit modifier que l'orientation de la diathèse et respecter les autres composantes du sens.

On dit parfois que le verbe utilisé au passif est la "copule", soit la forme du verbe être qui lie un nom à un attribut (par exemple Wilmet, 2000, 20003), et non un auxiliaire spécifique. Les arguments sont biaisés: on montre que le verbe être du passif n'est pas identique à l'auxiliaire temporel des verbes de mouvement (ceux qui ont être comme auxiliaire de passé. Il peut y avoir, pour être, une autre opposition, aspectuelle celle-là, qui distingue entre un processus en cours et un accompli. Cette opposition est rendue visible par le passif: par là, le passif se distingue du verbe construisant un attribut.

Le présent passif fait alors problème, parce que la même combinaison être+ Vpp est par ailleurs facilement interprétée comme statique et accomplie.

(12) Le mât est dressé sur la place

(13) Le mât est dressé sur la place à $8 h 45$ / par les ouvriers

Seule la seconde phrase est une variation de la forme active au présent, donc un véritable présent passif. Dans cette phrase, le verbe être ne 
présente pas une propriété, il participe à la description d'un processus en cours.

Le présent passif est souvent délaissé avec les verbes d'action et semble de généralisation tardive, si on en croit le grammairien Meigret. Meigret (1550) distingue l'aspect intrinsèque du verbe selon le critère de la "continuité" opposée à la "perfection et fin d'action". Un "continu" est un verbe comme "aimer" qui décrit un état; un "perfectif" comme blesser semble ne pas avoir à cette époque de présent passif. Je cite Meigret: "Je suis blessé ne se pourra résoudre par l'actif présent on me blesse: d'autant qu'étant l'action reçue, elle cesse".

Ce que décrit Meigret, c'est l'effet de l'interprétation accomplie, qui peut interférer avec l'interprétation temporelle précise du passif, parce que le verbe "être" est un vecteur imparfait de cette différenciation.

L'auxiliaire de passif au présent (ou à l'imparfait) a alors un sens spécifique qui désigne un état en devenir, "devient" avec les verbes qui imposent une modification de l'état de leur $2 \mathrm{e}$ actant, ou si on veut, sous forme de périphrase, "est en train d'être Vpp". Le présent passif reste cependant peu utilisé.

Il est important (malgré ce qu'on en dit quelquefois, cf. Gaatone, 1998: 29; Jalenques 2015) de distinguer l'auxiliaire du passif de la copule, c'est-àdire de l'emploi de être en construction attributive (Helland mais aussi un grammairien classique comme Bonnard). La distinction est nette entre un état et un procès:

(14) La route est goudronnée (/ par les employés municipaux et au passé composé, il y a une différence potentielle entre un état disparu et un temps du passé:

(15) La route a été goudronnée (, mais ça ne se voit plus)

(15') La route a été goudronnée il y a un mois (donc elle l'est)

L'interprétation d'un état est la plus immédiate, en particulier avec les participes passés à valeur ou emploi adjectival:

(16) Je suis fatigué

(17) La mer / la soupe est salée

Aucun agent extérieur n'est indispensable ici, et l'interprétation est celle d'une construction adjectivale. Le passif au sens "processif", avec une action au présent, reste possible:

(17') La mer est salée par l'action des rifts océaniques

On n'a pas en français l'opposition aspectuelle visible en allemand, entre auxiliaire du passif "werden" (devenir) et emploi du verbe être, "sein". Le passif requiert normalement "werden", même pour un verbe statif:

(18) Peter wird von Lise geliebt / ?? Peter ist von Lise geliebt 'Pierre est aimé de Lise'

mais on peut trouver les deux constructions, surtout quand une interprétation accomplie est possible: 
(19) Wir werden sehr oft eingeladen / wir sind sehr oft eingeladen 'Nous sommes très souvent invités'

Avec un agent, la règle reste l'auxiliaire spécifique, mais au temps composé on peut oublier 'worden' (participe passé de werden), et ne reste alors que le verbe sein au passé :

(20) Diese Mauer wurde von den Römern gebaut Ce mur devint par les Romains bâti

'Ce mur fut bâti par les Romains'

(21) Diese Mauer war von den Römern gebaut (worden)

Ce mur fut par les Romains bâti (devenu)

'Ce mur a / avait été bâti pâr les Romains'

Il y a donc en allemand une forme de passif d'état (passif de "Zustand") qu'on trouve quelquefois en français avec un agent:

(22) Elle était prévenue contre moi par tant d'amis communs (Skårup 1998)

$=$ Tant d'amis communs l'avaient prévenue contre moi

(23) Ces lettres sont écrites par des mains différentes (ibid.)

$=\quad$ Ces lettres ont été écrites par des mains différentes

(24) Tout était très bien arrangé par les maîtres de maison (ibid.)

$=\quad$ Les maîtres de maison avaient tout très bien arrangé

(25) La finale de The Voice ne se déroulera que le 12 mai. Pourtant, les jeux seraient déjà faits. Depuis des semaines, tout est prévu par la maison de disques qui produira le vainqueur. (programmetv-net $>$ The Voice, 6/5/2012)

$=$ tout a été prévu...

Ce sont en quelque sorte des constructions passives par l'agencement des actants, mais sans conjugaison passive dans le verbe, dans la mesure où le présent ou l'imparfait de l'auxiliaire est le temps actualisé, le participe et ce qui suit étant attributs au sens accompli.

2.2. Variantes pour être au passif.

Il arrive parfois qu'on trouve en français une sorte de passif avec "devient", rendant perceptible le processus :

(26) A quel point ma vertu devient-elle réduite? (Corneille, Horace,

(27) L'hypertexte devient transformé en un texte à tiroirs. (L. Allemand, J.M. Oullion, Les grandes questions des médias, coll. Les guides de l'étudiant) 
(28) ...en 2007, malgré un moratoire de quinze ans, il y avait encore moins de morues qu'en 1992; l'équilibre avait été rompu, les oufs des morues restantes étant systématiquement mangés par des espèces plus petites: le prédateur est devenu mangé par ses proies. (Alexandre Poussin, Marche avant, 2011)

De même avec être en train d'être:

(29) ...le contexte (...) se projette obligatoirement sur le texte qui est en train d'être dit (...) (B. Pottier cité par Gaatone, 1998, 18)

(30) Un nouveau pas en avant est en train d'être fait (Gaatone, ibid.)

\section{LE PASSIF IMPERSONNEL: UNE EXTENSION LARGE.}

Il y a deux diathèses distinctes qui se ressemblent en ce que toutes deux mettent en avant l'action verbale; le passif impersonnel se distingue parce que la relégation à l'arrière-plan du 1er actant laisse en position postverbale le 2e actant:

(31) Il a été utilisé une nouvelle méthode (par les chirurgiens) (Gaatone, 1998: 117)

Dans les conditions requises pour l'occurrence d'un clitique, on peut voir que le 2e actant direct postposé peut se pronominaliser comme un objet:

(32) Louis sera jugé par la Convention, ainsi qu'il l'a été décidé (Procès de Louis 16, vol.3, 1795)

(33) Comme il l'a été décidé en 2015, l'académie de Clermont partage aujourd'hui la zone A avec Lyon, Dijon, Besançon, Grenoble, Limoges, Poitiers et Bordeaux. (La Montagne, 30/8/2016)

D'une certaine façon, le 2e actant reste en place avec ses propriétés, comme cela se remarque avec les transitifs indirects:

(34) Il a été procédé à une distribution de couvertures

Les emplois sont largement ouverts aux intransitifs:

(35) Il a été beaucoup fumé dans cette salle

Le même problème d'interprétation entre action en cours et accompli existe, et ici aussi, le passif préfère les temps perfectifs:

(36) ??Il était dansé pendant des heures

(37) Il a été dansé toute la soirée

Il faut ajouter à cela des contraintes particulières, pas forcément aussi absolues qu'on l'a dit quelquefois:

- L'absence obligatoire de l'agent; contrainte rejetée par Gaatone:

(38) ?Il a déjà été dormi dans ce lit (par les plus illustres personnalités) (Gaatone, p.125)

Avec parler, il faut distinguer l'emploi intransitif du transitif indirect, qui permet l'agent:

(39) *Il a été parlé par tous les participants

(40) Il a déjà été parlé de cela par tous les participants

- L'obligation d'un complément quelconque (Gaatone)

(41) Il fut dansé, sauté, ballé. (La Fontaine, cité par Bonnard) 
Cet emploi n'est plus possible de nos jours: il faut un complément ou un adverbe quelconque pour ancrer la phrase dans une situation d'énonciation acceptable, peut-être pour rendre manifeste l'éloignement de la relation action à agent.

- L'agent doit être "humain".

Il faut peut-être distinguer ici la construction des transitifs de celle des intransitifs. Avec les transitifs, il doit être possible d'avoir l'agent sans contrainte de ce type:

(42) Il a été arraché une dizaine de toitures sous l'effet de la tempête (N Papon-Rivière 1995, 120)

On peut y opposer, pour les intransitifs, les emplois suivants de fumer:

(43) *Il a été fumé pendant des décennies dans cette vallée (par des cheminées d'usine)

(44) Il a été fumé pendant des heures dans ce couloir, ça empeste Les exemples demandent ici un agent "humain" (Gaatone 1998: 130) - Les verbes à auxiliaire temporel être ne sont pas utilisables au passif. Ces verbes sont généralement considérés comme impassivables en français, mais il semble que cela soit possible marginalement dans cette construction pour quelques verbes:

(45) Il a été retombé dans les mêmes erreurs (N. Papon-Rivière 1995:159)

(46) Il a été parvenu à un compromis acceptable (A. Zribi-hertz, 1987)

(47) Jamais encore il n'avait été descendu aussi bas (par aucune équipe au monde) (L. Tasmowski 1990)

Pour le présent passif, on constate les mêmes difficultés que pour le passif personnel. Le sens processif est possible:

(48) Il est procédé à une distribution de couvertures

Mais on trouve ici aussi le sens accompli, avec la copule:

(49) Il est maintenant prouvé qu'il a triché

$=\quad$ On a prouvé qu'il a triché

Le sens présent passif (On prouve maintenant qu'il a triché) est possible, mais peu vraisemblable.

\section{LE PASSIF "REFLEXIF" OU PRONOMINAL.}

Le terme "réflexif" de Damourette et Pichon permet d'éviter celui de réfléchi, qui est lié à l'interprétation d'une occupation par un même référent de deux positions actancielles distinctes.

Pour Gaatone (1998: 29), qui base le passif sur le participe passé, ce ne sont pas des passifs.

Pour d'autres, il existe bien un passif, plus souvent dénommé passif pronominal (cf. Lamiroy 1993, Herslund 2000); Herslund montre que la terminologie grammaticale est assez fluctuante sur ce point; pour Melis (1990), il s'agit de "médio-passifs". L'origine latine de l'interprétation 
passive à partir du réfléchi se ne fait aucun doute (Ernout \& Thomas, \$234), comme sa généralisation en latin tardif, par exemple dans :

(50) Mala rotunda...toto anno servare se possunt. (Palladius, $4 \mathrm{e}$ siècle)

'Les pommes rondes peuvent se conserver toute l'année'

La particule réfléchie peut signifier une confusion des rôles, soit l'interprétation "moyenne", sans distinction possible de l'agent et du patient:

(51) La branche se casse

On passe du "moyen" au passif quand le groupe nominal actant n'a pas du tout d'agentivité. L'agent, en principe non réalisé, est le plus souvent un "humain" indéterminé. On peut aussi supposer que le réfléchi autorise un sens proche du passif: N0 (agent) équivaut à N1 (patient), donc N0 se V peut signifier N0/N1 "devient" Vpp pour un verbe d'action.

Les langues romanes parcourent une sorte de cycle du réfléchi dont le français ne réalise que les premières étapes: celle du passif pronominal, puis celle du passif impersonnel pronominal limité aux verbes transitifs. L'italien réalise un cycle complet, dans une évolution qui est relativement récente $(\mathrm{du} 19 \mathrm{e}$ au milieu du 20e siècle selon Barbara Wehr). Cette évolution montre que la voix réflexive conduit à toutes sortes de hiérarchies des rôles actanciels, y compris parfois à des phrases avec agent (usage toscan) :

(52) Noi si andó / noi si verrà più tardi nous se va / nous se verra plus tard 'nous on s'en va / nous on se verra plus tard' (Wehr 1995: 245)

Cette interprétation peut se marquer par accord du participe passé :

(53) Noi ci si è bevuti una bira nous se se est bus (pl.m. ) une bière ('on s'est bu une bière') (Roegiest 2000: 161)

Le français a deux constructions passives pronominales: l'une avec sujet, l'autres impersonnelle.

4.1. Diathèse réflexive passive personnelle.

Toutes les deux sont surtout utilisées avec les verbes transitifs:

(54) Les pommes se mangent en toute saison

Le sens est souvent habituel, parfois prescriptif (c'est ce qui se fait), d'où son emploi dans les publicités:

(55) La télé couleur se loue chez Locatel (Herslund 2000)

Le français classique employait plus fréquemment cette construction, que connaissait déjà Meigret en 1550; le complément d'agent est normal à l'époque: 
(56) Ce vin se boit par les ivrognes (Meigret; inacceptable aujourd'hui)

(57) C'est une chose bien étonnante qu'elle (la loi) se soit toujours conservée si constamment durant tant de siècles par un peuple rebelle et impatient comme celui-ci. (Pascal, Pensées)

Les emplois actuels avec complément d'agent sont devenus bien plus rares, mais existent.

(58) L'hébreu se parle et s'écrit tout le long du jour en Palestine par les enfants, leurs parents et leurs grands-parents. (cité par Herslund 2000: 74)

En voici des exemples récents :

(59) La zoothérapie se pratique par des professionnels spécialisés.. (agatea.org, 17/10/08)

(60) Le rugby est un sport qui se pratique par des personnes vêtues de vêtements super-moulants afin de mettre en évidence leur surcharge pondérale manifeste. (désencyclopedie.wikia.com)

(61) L'approche de la forêt landaise s'annonce par un paysage compartimenté en clairières qui forme une transition à l'ouest de Casteljaloux. (atlaspaysages.lotetgaronne.fr)

Souvent, on trouve un instrumental plutôt qu'un agent:

(62) Les élèves se recrutent sur concours, sur épreuves pour les écoles d'enseignement technique ou sur titre pour les écoles préparatoires. (legifrance.gouv.fr, 13/2/17)

\subsection{Diathèse réflexive passive impersonnelle.}

Elle est utilisée avec des transitifs, laisse en place l'objet et aboutit parfois au sens "on" pour l'agent (verbes "de parole", il se dit que...). Avec ce sens: il se murmure, il se répand, il se chuchote, il se répète que...)

(6) Il se raconte de drôles d'histoires sur ton compte

La construction était plus ouverte aux intransitifs que de nos jours, en français classique:

(63) Il ne se parlera plus de tous ces faits éclatants (Bossuet, cité par Grevisse, §1400)

En français actuel, on ne les trouve plus guère. N. Rivière propose:

(64) ?Il se bouquine plus sur les quais que dans les supermarchés (N.Papon-Rivière, p.458)

Les verbes sont des verbes de parole ou d'activité dans lesquels l'agent importe peu: ils sont orientés vers l'action présentée souvent comme une somme ou un bilan:

(65) Il s'est vendu 5,5 millions de voitures d'occasion en 2016, contre seulement 1,9 million de voitures neuves. (huffingtonpost.fr, 27/4/17)

(66) Comme prévu, il s'est échangé moins de SMS pour les vœux de 2016. (frandroid.fr, 1/1/16) 
(67) En 2014, en France, il s'est bu 49 litres de jus de fruits...par seconde. (laperofermier.fr, 2015)

La difficulté est parfois de séparer le sens moyen du passif; en (68), le sens pourrait être moyen, le paquebot participant à sa perte. Le sens passif suppose un agent humain : "on perdait"...

(68) Quelqu'un disait, ce soir, que d'après des relevés de statistiques paraissant très exacts, il se perdait par jour un paquebot à vapeur sur les océans de ce monde...(Goncourt, III, 634, cité par N. Papon, 456)

Dans l'exemple suivant, de façon caractéristique, c'est un peu comme si le processus se réalisait seul, sans nécessité d'un agent extérieur:

(69) Progressivement, il se révèle que ce village lacustre était au centre d'un vaste réseau d'échanges qui traversait toute l'Europe. (Le Monde, 24/6/17, p.18)

\section{INFINITIF ET PASSIF. CONSTRUCTIONS AVEC $A$ VINF.}

Si le critère à retenir n'est pas la présence du participe passé, comme le propose Gaatone, on peut regarder du côté de l'infinitif qui a des orientations de diathèse qui peuvent cibler le $2 \mathrm{e}$ actant.

1) Il y a d'abord les passifs complexes (Damourette et Pichon) à auxiliaire d'aspect, comme finir, achever ou commencer. On obtient parfois un passif qui englobe l'ensemble de la construction:

(70) Le château n'était pas achevé de meubler (Chateaubriand cité par H. Bonnard)

Le passif sur l'infinitif est exclu:

(70') *Le château n'achevait pas d'être meublé

Mais parfois le passif est aussi construit sur l'infinitif:

(71) (Le bâtiment) n'était pas achevé d'être bâti... (Flaubert, Madame Bovary, dans Sandfeld 1965:15)

L'extension du marquage passif d'un premier verbe, l'infinitif restant non modifié, était plus large dans l'ancienne langue, qui disait

(72) Un homme est prêt à pendre (Bouhours, 17e s., Haase §85)

Cet emploi existe encore pour certains verbes:

(73) Ce vin est prêt à mettre en bouteilles

2) Il y a aussi la possibilité de trouver l'infinitif après la construction à auxiliaire se Vperception, où l'infinitif alterne parfois avec le participe passé:

(74) Comme il atteignait l'Olympia, il se vit dépassé par une dame assez épaisse... (Morand, cité par Sandfeld, p. 185)

(75) Il se sentit envahir par un sentiment de peur (ibid. 184) $\mathrm{Y}$ a-t-il toujours une différence entre l'emploi de l'infinitif et celui du participe? La participe passé serait plutôt lié à l'accompli, et l'infinitif à l'aspect processif ou cursif (J. François 2003). Le verbe le plus malléable pour cette variation semble être se voir (François, 2003: 285) considéré 
parfois (Bat-Zeev) comme un auxiliaire de substitution, aussi bien pour le passif de 2e actant que pour le passif de bénéficiaire. Avec des limites, puisqu'on ne peut pas le dire pour tout infinitif:

(76) *Il s'est vu écraser / Il s'est fait écraser (Bat-Zéev)

3) La construction attribut être à Vinf constitue une sorte de pseudo-passif prospectif qui complète la version accomplie avec être Vpp:

(77) Ce qui est fait n'est plus à faire

Le sens est le plus souvent orienté vers le futur et une valeur modale de nécessité:

(78) Le sèche-mains est à réparer par la mairie.

(14/3/17, ecole.elementaire.anbord)

(79) Toute dégradation volontaire mineure est à réparer par le participant. (baroudeurs.be, 3/2017)

Sans auxiliaire:

(80) A faire par les amateurs de BD. (tripadvisor.fr, 8/4/17)

Le sens futur, ou une sorte de présent d'attente, est très fréquent:

(81) La cristallerie Baccarat est à vendre par le propriétaire Starwood (10/9/09) (= est mise en vente)

Y a-t-il aussi un présent, ce qui renforcerait le parallélisme avec le passif à participe passé? Comme pour le passif régulier, le présent processif n'est pas très fréquent avec $\grave{a} \operatorname{Vinf}$. On trouve des exemples qui parfois font penser à des constructions neutres:

(82) J'ai suivi les instructions mais probablement trop mouillé mon matelas. Voilà maintenant $24 \mathrm{~h}$ qu'il est à sécher et cela ne s'améliore guère. (toutpratique.com, 3/1/16)

On peut dire quand même avec un locatif qui ressemble à un agent:

(83) Ce costume était à nettoyer chez le teinturier

(84) Ma voiture est à réparer depuis ce matin chez le garagiste ce qui se rapproche du présent passif:

(84') (?)Ma voiture est réparée depuis ce matin par le garagiste La ressemblance est nette, mais l'un est nettement orienté vers le passé, l'autre vers le futur: la difficulté de réaliser un présent passif subsiste donc. En somme, autant le passif à participe passé des verbes d'action tire vers l'accompli, autant la construction avec à Infinitif des mêmes verbes tire vers le non encore accompli, l'interprétation processive étant difficile dans les deux cas.

La correspondance la plus nette est donc celle entre le passif version accompli et cette forme prospective qui en est l'opposé ou le complémentaire pour les verbes d'action.

\section{CONCLUSION}

La construction verbale au passif utilise divers outils du mot verbal étendu (incluant auxiliaires et clitiques pronominaux), depuis les positions clitiques avec $i l$ et $s e$ et l'auxiliaire être qui peut manifester si nécessaire 
un sens processif, jusqu'au participe passé, parfois jusqu'à l'infinitif; l'infinitif dans sa version avec $a ̀$ réalise une sorte de passif prospectif qui est le pendant du passif de Zustand utilisant la copule. Aucune des constructions examinées n'est mécaniquement utilisable pour produire un passif adapté à un verbe quelconque. La construction verbale qui a le plus d'extension et de généralité, malgré ses défaillances, reste la construction associant être et le participe passé, et le sens processif de être, distingué du sens accompli, reste le marqueur le plus spécifique du passif dans le verbe. Le passif pronominal n'a aucun problème avec le sens processif, mais d'une part l'agent est souvent difficile à construire, d'autre part, sa version impersonnelle dérive soit vers une signification agentive indéterminée de type "on", soit vers une interprétation de type "moyen", où c'est le processus verbal qui semble se réaliser seul.

$\mathrm{Au}$ total, le passif n'est pas une construction totalement grammaticalisée en français, comme dans la plupart des langues.

Claude MULLER ${ }^{3}$

\section{RÉFÉRENCES BIBLIOGRAPHIQUES}

BAT-ZEEV, H. 19970. «La grammaticalisation des auxiliaires: le cas de voir ». Scolia 10, 205-224.

BONNARD, H. 1976. "Passif». Article du Grand Larousse de la Langue Française.

DAMOURETTE, J., E. PICHON 1911-1940. Des mots à la pensée, Essai de grammaire de la langue française. D'Artrey, Paris.

ERNOUT, A., F. THOMAS 1953 (2002). Syntaxe latine. Klincksieck, Paris.

FRANÇOIS, J. 2003. La prédication verbale et les cadres prédicatifs. Peeters, Louvain.

GAATONE, D. 1998. Le passif en français. Duculot, Bruxelles.

GREVISSE, M. (1980). Le bon usage. Duculot, Bruxelles.

HAASE, A. 1965. Syntaxe française du XVIIe siècle. Delagrave, Paris.

HELLAND, H.P. 2001. Le passif périphrastique en français. Museum Tusculanum Press, Univ. of Copenhagen, Copenhague.

HERIAU, M. 1980. Le verbe impersonnel en français moderne. Presses Universitaires de Lille.

HERSLUND, M. 2000. «Les deux passifs du français ». In Schøsler L. (éd), 71 81.

JALENQUES, P. 2015. «Le passif en français et les lacunes distributionnelles des verbes ». Corela 13-1, URL : http://corela.revues.org/4015.

LAMIROY, B. 1993. " Pourquoi il y a deux passifs ». Langages, 109, 53-72.

MEIGRET, L. 1550. Le tretté de la grammere françoeze. Slatkine reprints, Genève.

3 Professeur émérite à l'Université Bordeaux Montaigne, membre de l'équipe CNRS CLLE-ERSS, UMR 5263. 
MELIS, L. 1990. La voie pronominale. Duculot, Bruxelles.

MULLER, C. 2000. "Le passif processif et ses concurrents. définition et quelques particularités. In: L. Schøsler (éd), 49-69.

-. 2007. «L'évolution cyclique du réfléchi: le passif pronominal dans le domaine roman ». In Rousseau A., Bottineau D. \& Roulland D. (éds): L'énoncé réfléchi, Presses Universitaires de Rennes, 187-202.

-. 2008². Les bases de la syntaxe. Presses Universitaires de Bordeaux.

PAPON-RIVIERE, N. 1995. Repères temporels et repères énonciatifs: la construction impersonnelle, le pronominal et le participe passé adjectival en français. Septentrion, Lille.

RENZI, L. \& SALVI, G. 1991. Grande grammatica italiana di consultazione, vol. III. Il Molino, Bologne.

ROEGIEST, E. 2000. «Pasiva refleja en espagnol et si passivante en italien: deux points de vue sur la même réalité ». In Coene M., De Mulder W., Dendale P., D'Hulst Y. (éds): Traiani Augusti vestigia pressa sequamur. Studia Linguistica in honorem Lilianae Tasmowsky. Unipress, Padoue, 155167.

SANDFELD, K. 1936 (1977). Syntaxe du français contemporain, L'infinitif. Droz, Genève.

SCHØSLER, L. (2000). Le passif, Actes du colloque international, Etudes Romanes, 45. Edité par L. Schøsler, Museum Tusculanum Press, Copenhague.

SHIBATANI, M. 1985. "Passives and related constructions: a prototype analysis ». Language 61, 821-848.

SKÅRUP, P. 1998. "Les emplois de être + participe passé en français contemporain ». In Bilger M., van den Eynde K., Gadet F. (éds): Analyses linguistiques et approches de l'oral, Recueil d'études offert en hommage à Claire Blanche-Benveniste, Peeters, Orbis supplementa, Leuven, 257-265.

TASMOWSKY, L. 1990. "...en semble ou semble en... ». Le Français moderne $58,28-43$.

WEHR, B. 1995. SE-Diathese im Italienischen. Gunter Narr, Tübingen.

WILMET, M. 2000. « Du passif à la topicalisation ou pour changer de sujet ». In: L. Schøsler (éd), 265-275.

-. $2003^{3}$. Grammaire critique du français. Duculot, Bruxelles.

ZRIBI-HERTZ, A. 1987. "La réflexivité ergative en français moderne». Travaux de linguistique et de philologie 32, 131-148. 